
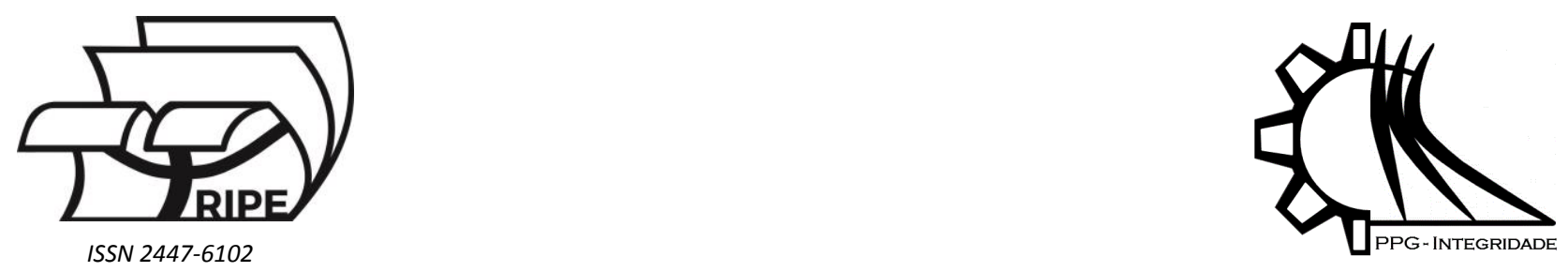

Article

\title{
Análise da discretização temporal para a simulação numérica de um espectro de ondas do tipo Pierson - Moskowitz
}

\author{
Luciano Rodrigues ${ }^{1, *}$, Ana Paula Del Aghenese ${ }^{2}$, Elizaldo Domingues dos Santos ${ }^{3}$, Liércio André Isoldi ${ }^{4}$, Luiz Alberto \\ Oliveira Rocha ${ }^{5}$, Mateus das Neves Gomes ${ }^{6}$ \\ 1, * Universidade Federal do Rio Grande - FURG; luciano.drigues@hotmail.com \\ 2 Universidade Federal do Rio Grande - FURG; anapauladelaghenese@hotmail.com \\ 3 Universidade Federal do Rio Grande - FURG; elizaldosantos@furg.br \\ 4 Universidade Federal do Rio Grande - FURG; liercioisoldoi@furg.br \\ 5 Universidade do Vale dos Sinos - UNISINOS; laorocha@gmail.com \\ 6 Instituto Federal do Paraná - IFPR Campus Paranaguá; mateus.gomes@ifpr.edu.br
}

Received: 14/12/2018; Accepted: 05/01/2019; Published: 29/01/2019

Resumo: $O$ presente trabalho apresenta uma metodologia para a simulação numérica de espectro de ondas em tanques. O objetivo é obter recomendações sobre a discretização temporal. Para tanto, o espectro de ondas de Pierson-Moskowitz utilizado no presente estudo possui algumas características como altura significativa da onda $\left(H_{s}\right)$ de 1,5 m e período significativo da onda $\left(T_{s}\right)$ de 7,5 $\mathrm{s}$. O domínio computacional é bidimensional e representa um tanque de ondas numérico. Além disso, uma importante atualização no modelo computacional é a eliminação da reflexão das ondas através de uma região de amortecimento, chamada de praia numérica. Para a solução numérica foi empregado um código de dinâmica dos fluidos computacional, FLUENT ${ }^{\circ}$ baseado no Método de Volumes Finitos (MVF). A geometria e a geração da malha são desenvolvidas no software GAMBIT'. O modelo multifásico Volume of Fluid (VOF) é aplicado no tratamento da interação água-ar. Os resultados obtidos indicam que é possível reproduzir numericamente a propagação de espectro de ondas em tanques com acurácia, sendo este um avanço em representar os fenômenos oceânicos de forma mais realística. Com isto, esses resultados poderão ser utilizados em trabalhos futuros, principalmente no estudo numérico de dispositivos conversores de energia das ondas do mar em energia elétrica, como por exemplo, os dispositivos de coluna de água oscilante (OWC) e de galgamento.

Palavras-chave: Tanque de ondas, Volume of Fluid, Espectro de Ondas, Praia Numérica.

\section{Analysis of temporal discretization for the numerical simulation of a Pierson - Moskowitz type wave spectrum}

\begin{abstract}
The present work presents a methodology for the numerical simulation of wave spectrum in tanks. The objective is to obtain recommendations on temporal discretization. To that end, the Pierson-Moskowitz wave spectrum used in the present study has some characteristics such as significant wave height (HS) of $1.5 \mathrm{~m}$ and significant wave period (TS) of $7.5 \mathrm{~s}$. The computational domain is two-dimensional and represents a number-wave tank. In addition, an important update in the computational model is the elimination of the reflection of the waves through a region of cushioning, called numeric beach. For the numerical solution, a computational fluid dynamics code, FLUENT ${ }^{\circ}$, was used based on the Finite Volume Method (MVF). Geometry and mesh generation are developed in GAMBIT ${ }^{\circ}$ software. The Multiphase Volume of Fluid (VOF) model is applied in the treatment of water-air interaction. The results indicate that it is possible to reproduce numerically the propagation of wave spectrum in tanks with accuracy, which is an advance in representing the ocean phenomena in a more realistic way. Therefore, these results can be used in future work, mainly in the numerical study of sea wave energy converters in electrical energy, such as oscillating water column (OWC) and overtopping devices.
\end{abstract}


Keywords: Wave Tank, Volume of Fluid, Wave Spectrum, Numerical Beach.

\section{Introdução}

A propagação de ondas de água de gravidade superficial é um fenômeno físico importante no campo da engenharia costeira e oceânica. As ondas podem ser alteradas pelo corte de ar, refração, difração e reflexão à medida que as ondas incidentes se propagam de regiões marinhas para regiões próximas da costa. Além disso, as ondas ao atingir uma zona próxima da costa podem causar erosões, além de comprometer a segurança das estruturas costeiras. Portanto, uma simulação precisa e eficiente da propagação de onda é crucial para uma melhor compreensão de tal fenômeno dependente do tempo (Zhang et al., 2006).

A necessidade de encontrar uma alternativa sem comprometer a integridade dos resultados levou ao desenvolvimento de tanques de ondas numéricas, uma representação numérica do tanque de ondas físicas. 0 desenvolvimento e o avanço no poder de processamento de computadores abriram caminho para o uso de códigos de Dinâmica dos Fluidos Computacionais (CFD) que são usados para realizar esta tarefa. Os tanques de onda ou os fluxos de ondas foram utilizados há décadas para realizar testes e pesquisas, fornecendo resultados muitos valiosos que ajudaram na concepção de dispositivos, estruturas, até mesmo ajudam os códigos de configuração (Prasad et al., 2017).

Muitas ferramentas foram implementadas nestes modelos nos últimos anos com o intuito de reproduzir tanques de ondas numéricos, análogos aos tanques de ondas de laboratórios físicos. Recentemente, alguns poucos modelos permitem a imposição de ondas irregulares, obtendo-se, desta forma, resultados mais próximos da realidade (Lisboa et al., 2016).

O conceito de onda irregular pode ser representado através de um espectro das ondas. Um espectro de ondas pode ser decomposto, por meio de análise harmônica (ou Fourier), em um grande número de ondas sinusoidais (regulares) de diferentes frequências, direções, amplitudes e fases. Onde, cada frequência e direção descrevem uma componente de onda, e cada componente possui uma amplitude e fase associadas. A análise harmônica fornece assim uma aproximação à forma irregular, mas quase periódica, de um registro de onda como a soma das curvas sinusoidais, resultando uma elevação da superfície variando no tempo em uma única direção (WMO, 1998).

Neste sentido, ao simular um fenômeno em que o modelo estiver mais próximo da realidade, mais detalhados e confiáveis serão os resultados e uma forma para que isto ocorra é através da atualização do modelo, em considerar a propagação de espectro de ondas juntamente com a técnica da praia numérica.

Tratando-se de espectro de ondas, é possível encontrar na literatura trabalhos que tem como objetivo a simulação numérica considerando espectro como, por exemplo, Elangovan e Sahoo (2010), Liang et al. (2010), Elangovan (2011), Cho et al. (2015), Costa et al. (2016), Lisboa et al. (2016), Gomes et al. (2016), Gatin et al. (2017) e Rodrigues et al. (2017). Além disso, foram encontrados estudos considerando tanque de ondas para a simulação numérica, entre estes, destaca-se Boo (2002), Park et al. (2004), Anbarsooz et al. (2013), Kim et al. (2014), Kamath et al. (2015), Finnegan e Goggins (2015), Vyzikas et al. (2017), Rezanejad et al. (2017) e Prasad et al. (2017).

Este trabalho tem como objetivo, através da simulação numérica em tanque de ondas, obter uma recomendação para a discretização temporal, que é uma atualização do modelo numérico para considerar um espectro de ondas, de Pierson-Moskowitz. Além disso, é considerada uma técnica de amortecimento da onda chamada praia numérica. Um avanço na representação computacional realística em tanques de ondas de fenômenos ligados ao oceano. São apresentadas verificações do espectro de onda com: período significativo da onda $\left(T_{s}\right)$ de 7,5 s e altura significativa da onda $\left(H_{s}\right)$ de 1,5 m. Na solução numérica é empregado um código de dinâmica dos fluidos computacional (CFD), baseado no Método de Volumes Finitos (MVF) e o modelo multifásico Volume of Fluid (VOF) para a interação água-ar.

\section{Representação Computacional}

\subsection{Modelagem física do problema}

O problema proposto consiste em analisar numericamente a propagação de um espectro de onda Pierson Moskowitz em tanque de ondas. A realização da simulação numérica da propagação do espectro de ondas requer o conhecimento das características da onda a ser gerada, assim é possível dimensionar o domínio computacional e posteriormente discretizar o domínio computacional. E com a aplicação das condições de contorno resolver as equações de conservação do modelo numérico. 
Uma atualização no modelo computacional foi realizada em respeito a eliminação da reflexão das ondas através de uma região de amortecimento, a praia numérica. Possibilitando assim, simulações com tempos reais maiores e tempos de CPU menores, semelhante ao desenvolvido em Lisboa et al. (2016).

O domínio computacional é dividido em regiões, conforme Fig. 1: i) região da propagação da onda e ii) região de absorção da onda (praia numérica). O comprimento total do tanque de ondas é de $C_{T}=3,5 \cdot \lambda_{s}$, que se dá através da soma das regiões $L_{1}=1,5 \cdot \lambda_{s}$ e $L_{2}=2,0 \cdot \lambda_{s}$, sendo $\lambda_{s}$ o comprimento significativo do espectro de ondas (m) referente ao período significativo, semelhante ao que foi realizado em Lisboa et al. (2016).

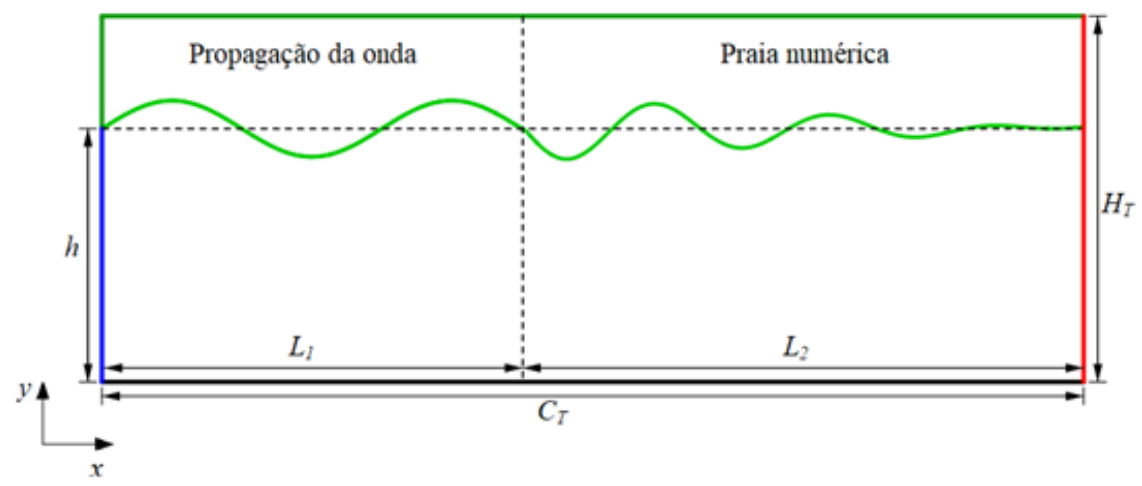

Figura 1. Representação esquemática do domínio computacional.

Em relação à altura total $\left(H_{T}\right)$ do tanque de ondas $(\mathrm{m})$, foi considerada a profundidade mais três vezes a altura significativa do espectro, como foi considerado $h=10 \mathrm{~m}$, assim, resulta em $H_{T}=14,5 \mathrm{~m}$, onde $h$ é a profundidade de propagação $(\mathrm{m})$.

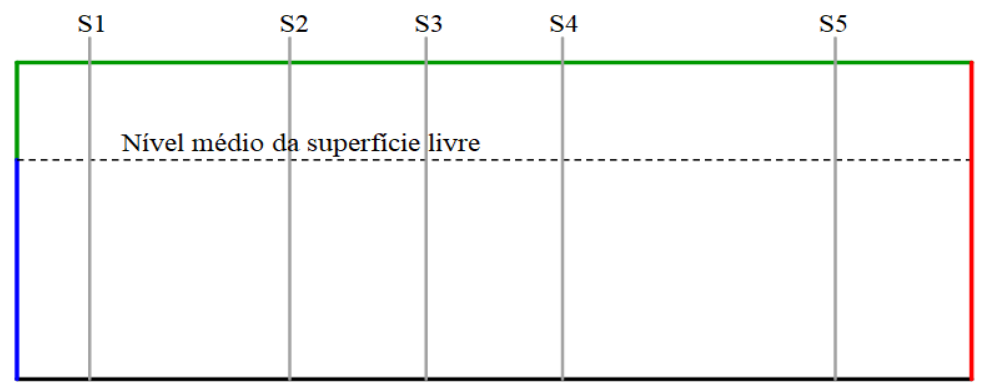

Figura 2. Representação esquemática do tanque de ondas.

Para o estudo e comparação da propagação do espectro, na Fig. 2, são impostas cinco sondas verticais de medição da elevação da superfície livre ao longo do tanque de ondas, nas seguintes posições: $\mathrm{S} 1\left(x=0,266 \cdot \lambda_{s} \mathrm{~m}\right)$, $\mathrm{S} 2\left(x=1,0 \cdot \lambda_{s} \mathrm{~m}\right), \mathrm{S} 3\left(x=1,5 \cdot \lambda_{s} \mathrm{~m}\right)$, S4 $\left(x=2,0 \cdot \lambda_{s} \mathrm{~m}\right)$ e S5 $\left(x=3,0 \cdot \lambda_{s} \mathrm{~m}\right)$. Vale lembrar que $\lambda_{s}$ representa o comprimento significativo da onda conforme o comprimento da onda, que pode ser observado na Tab. 1.

O comprimento do espectro adotado é referente ao período das componentes do espectro. Na Tab. 1, têm-se as características do espectro de ondas, onde é possível observar que tanto o comprimento significativo quanto o período significativo do espectro são inversamente proporcionais em relação à frequência significativa, pois está se tratando de frequências no âmbito do espectro. Portanto, o que ocorre é que a frequência mínima vai conduzir a um período maior e uma frequência maior a um período menor, que é calculado pela Eq. (3.13). 
Tabela 1. Características do espectro de ondas

\begin{tabular}{cccc}
\hline Características & Mínimo & Pico & Máximo \\
\hline Frequência (rad/s) & $\omega_{\text {mín }}=0,5$ & $\omega_{P}=0,84$ & $\omega_{\text {máx }}=3,0$ \\
Período $(\mathrm{s})$ & $T_{\text {mín }}=12,57$ & $T_{P}=7,50$ & $T_{\text {máx }}=2,09$ \\
Comprimento $(\mathrm{m})$ & $\lambda_{\text {mín }}=119,0$ & $\lambda_{P}=65,35$ & $\lambda_{\text {máx }}=6,84$ \\
\hline
\end{tabular}

\subsection{Geração do espectro Pierson - Moskowitz}

O espectro de Pierson - Moskowitz é válido para um mar totalmente desenvolvido, ou seja, é o estado do mar onde desenvolvem-se ondas máximas correspondentes à força do vento atuante, e assume que as ondas estão em equilíbrio com o vento (Chakrabarti, 2005).

Um fluxograma do processo da geração e da análise de ondas irregulares, representada pelo espectro de Pierson - Moskowitz é apresentado na Fig. 3.

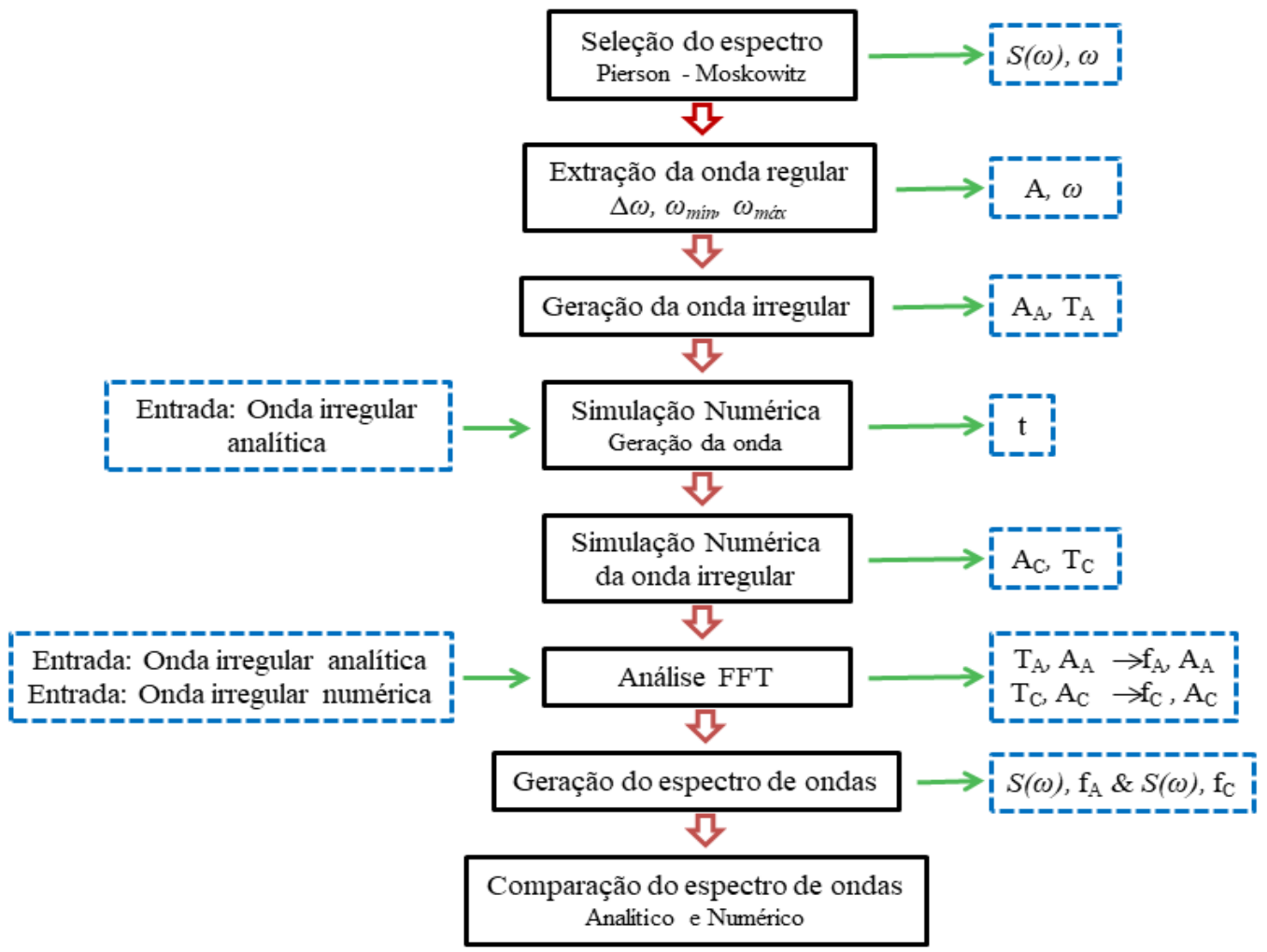

Figura 3. Metodologia de análise da geração de ondas irregulares. Adaptado de Elangovan (2011).

Assumindo um mar totalmente desenvolvido (produzido por ventos soprando constantemente durante centenas de milhas por vários dias) submetido a um espectro de onda, Pierson - Moskowitz, a densidade espectral ( $S(\omega))$ é dada por (Elangovan, 2011):

$$
S_{P M}(\omega)=\frac{5}{16} \frac{H_{S} \omega_{P}^{4}}{\omega^{5}} e^{-\left(\frac{5 \omega_{P}^{4}}{4 \omega^{4}}\right)}
$$

onde: $H_{S}$ é a altura significativa $(\mathrm{m}), \omega$ é a frequência da onda ( $\left.\mathrm{rad} / \mathrm{s}\right)$ e $\omega_{P}$ é a frequência de pico da onda $(\mathrm{rad} / \mathrm{s})$ e pode ser determinada por:

$$
\omega_{P}=\frac{2 \pi}{T_{S}}
$$


onde: $T_{S}$ é o período significativo do espectro (s).

Sendo que, neste trabalho, foram considerados $H_{S}=1,5 \mathrm{~m}$ e $T_{S}=7,5 \mathrm{~s}$, conforme foram empregados também em Elangovan (2011) e em Gomes et al. (2016).

De acordo com ANSYS (2016), é possível determinar o intervalo de frequências de modo que o compreenda a região de maior energia da onda, por:

$$
\begin{aligned}
& \omega_{\text {min }}=0,5 \cdot \omega_{P} \\
& \omega_{\text {máx }}=3,0 \cdot \omega_{P}
\end{aligned}
$$

Neste estudo, o espectro é formado por 15 ondas com frequências dentro do intervalo determinado pelas Eqs. (3) e (4). Assim, é possível distribuir de forma igual às frequências a serem consideradas através da seguinte relação:

$$
d \omega=\frac{\left(\omega_{\operatorname{máx}}-\omega_{\min }\right)}{n}
$$

onde: $n$ é o número de ondas consideradas no espectro.

É possível extrair cada uma das 15 ondas regulares que compõem o espectro de ondas considerado. Para tanto deve ser considerada a seguinte relação entre densidade espectral e amplitude (Elangovan, 2011):

$$
S\left(\omega_{n}\right) d \omega=\frac{1}{2} \zeta_{a}^{2}
$$

onde: $\zeta_{a_{n}}$ é a amplitude da componente do espectro $(\mathrm{m})$, ou seja, a amplitude de uma das 15 ondas que formam o espectro.

Mantendo o ângulo de fase diferente para cada onda regular, a onda irregular pode ser gerada analiticamente conforme apresentado a seguir (Elangovan, 2011):

$$
\zeta(t)=\sum_{n=1}^{n_{0}} \zeta_{a_{n}} \cos \left(k_{n} x-\omega_{n} t+\varepsilon_{n}\right)
$$

onde: $k_{n}$ é o número de onda da componente $(\mathrm{rad} / \mathrm{m}), x$ a posição espacial $(\mathrm{m}), \omega_{n}$ a frequência da componente (rad/s), $t$ o tempo (s) e $\varepsilon_{n}$ o ângulo de fase randômico da componente (rad).

O número de onda pode ser determinado pela relação de dispersão, dada por (Dean e Dalrymple, 1991):

$$
\omega^{2}=g k \tanh (k h)
$$

onde: $g$ é a aceleração da gravidade $\left(\mathrm{m} / \mathrm{s}^{2}\right)$ e $h$ é a profundidade $(\mathrm{m})$.

A geração numérica do espectro Pierson-Moskowitz considera as componentes do espectro extraídas a partir da Eq. (7). Após, a onda irregular numérica gerada passa por uma análise através da Transformada Rápida de Fourier (FFT - Fast Fourier Transform) para mudar o espaço da análise, ou seja, é utilizada para remontar o espectro da densidade numérica, elevando os dados de um campo (elevação por tempo) para o campo da frequência (densidade por frequência) e assim realizar a comparação com a densidade espectral analítica.

Na Figura 4(a) é realizada a decomposição do espectro, apresentando algumas ondas regulares representativas do espectro em consideração, onde são apresentadas cinco ondas regulares de diferentes frequências: onda 1 com $\omega_{1}=0,583 \mathrm{rad} / \mathrm{s}$, onda $3 \mathrm{com} \omega_{3}=0,917 \mathrm{rad} / \mathrm{s}$, onda $5 \mathrm{com} \omega_{5}=1,25 \mathrm{rad} / \mathrm{s}$, onda $10 \mathrm{com} \omega_{10}=2,083$ $\mathrm{rad} / \mathrm{s}$ e onda $15 \mathrm{com} \omega_{10}=2,917 \mathrm{rad} / \mathrm{s}$. Sendo a frequência de pico do espectro, $\omega_{P}=0,837758 \mathrm{rad} / \mathrm{s}(\mathrm{Eq}$. (2)), é possível observar que quando a frequência de uma componente estiver mais próxima da frequência de pico, maior é a predominância da onda no espectro.

Na Figura 4(b) é apresentado a elevação da superfície livre formada pelo somatório das quinze ondas regulares, com o objetivo de evidenciar o efeito do somatório de diversas ondas com diferentes frequências (Eq. (7)). 

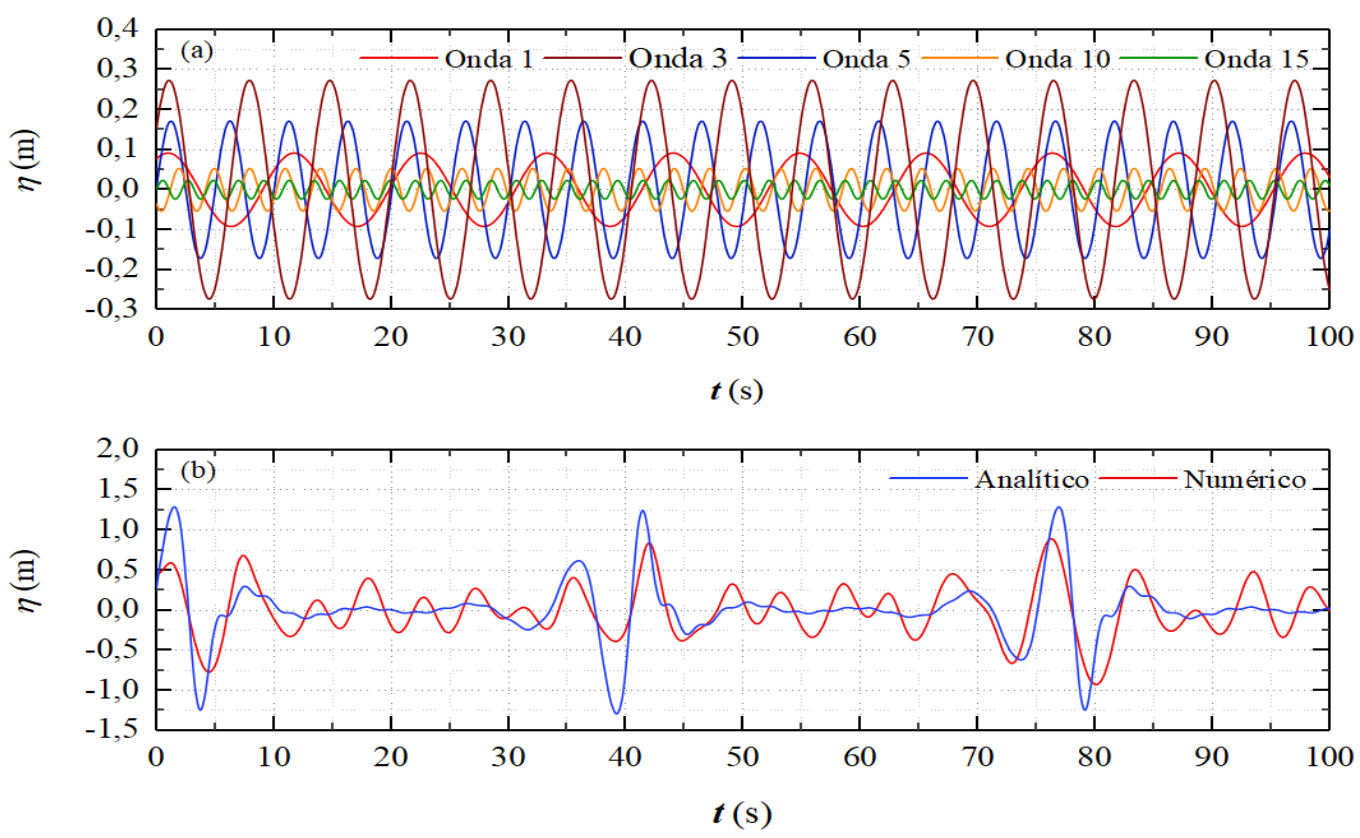

Figura 4. (a) Decomposição do espectro em ondas regulares e (b) Elevação da superfície livre.

Cabe destacar que foram consideradas 15 componentes de ondas, o avanço no tempo de 0,01 s, profundidade (h) de $10 \mathrm{~m}$, comprimento significativo do espectro de ondas de $119 \mathrm{~m}$ e a sonda de monitoramento em $x=0,2667$. $\lambda_{S} \mathrm{~m}$, ou seja, na posição de $31,7 \mathrm{~m}$.

\subsection{Condições de contorno}

Conforme o indicado na Fig. 5, o gerador de ondas está posicionado na parte inferior da superfície lateral esquerda do tanque de ondas, com a condição de contorno de velocidade de entrada prescrita. Para a geração de ondas irregulares é considerada a Eq. (7) e suas respectivas componentes, horizontal $(u)$ e vertical $(w)$, da velocidade da onda como condição de contorno. Essas componentes da velocidade variam em função do espaço e do tempo e são impostas na superfície de entrada e dadas por (Dean e Dalrymple, 1991):

$$
\begin{gathered}
u=\zeta_{a_{n}} g k_{n} \frac{\cosh \left(k_{n} z-k_{n} h\right)}{\omega_{n} \cosh \left(k_{n} h\right)} \cos \left(k_{n} x-\omega t\right) \\
w=\zeta_{a_{n}} g k_{n} \frac{\operatorname{senh}\left(k_{n} z-k_{n} h\right)}{\omega_{n} \operatorname{senh}\left(k_{n} h\right)} \operatorname{sen}\left(k_{n} x-\omega t\right)
\end{gathered}
$$

onde: $\omega_{n}$ é a frequência da componente, e $z$ é a variação da posição entre a superfície livre da água e o fundo do $\operatorname{mar}(m)$.

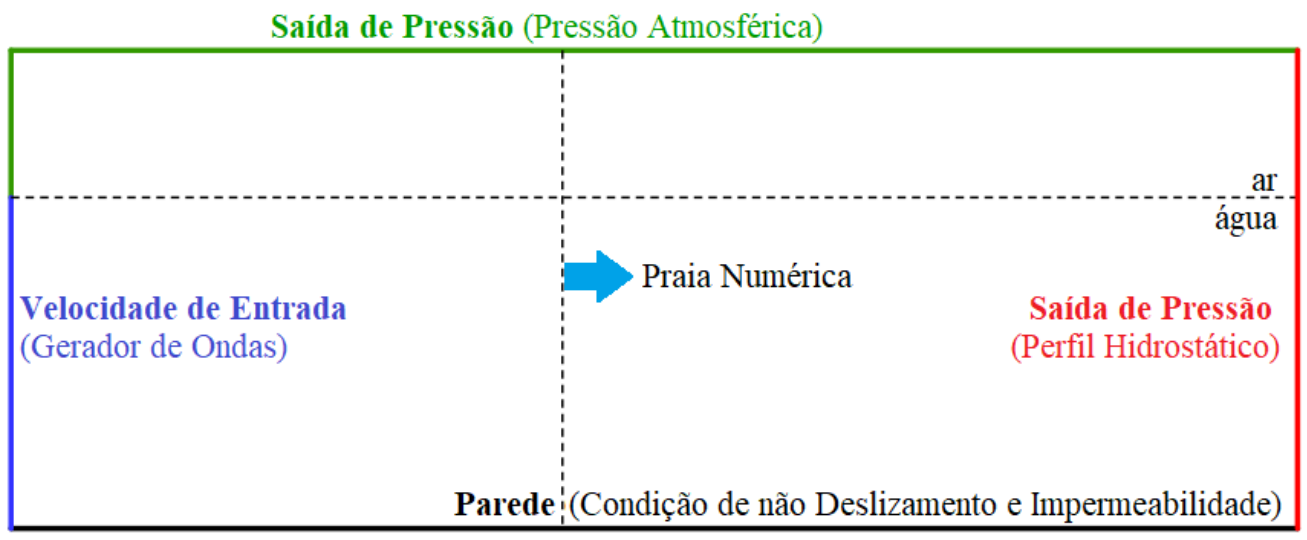

Figura 5. Representação esquemática das condições de contorno. 
Com relação às demais condições de contorno, na parte superior da superfície lateral esquerda e na superfície superior do tanque é aplicada uma condição de contorno de pressão atmosférica. Na superfície inferior do tanque uma condição de não-deslizamento e impermeabilidade com velocidade nula e na superfície lateral direita é imposta a condição de pressão hidrostática.

Para evitar a reflexão da onda, na região de amortecimento é adicionado um termo sumidouro $(S)$ na equação da conservação da quantidade de movimento, a fim de realizar o amortecimento da onda incidente próximo ao limite da saída de pressão. Conforme (Lisboa et al., 2016; Fluent, 2017):

$$
S=-\left[C_{1} \rho V+\frac{1}{2} C_{2} \rho|V| V\right]\left(1-\frac{Z-Z_{f s}}{Z_{b}-Z_{f s}}\right)\left(\frac{x-x_{s}}{x_{e}-x_{s}}\right)^{2}
$$

onde $C_{1}$ e $C_{2}$ são, respectivamente, coeficiente de amortecimento linear (1/s, resistência por unidade de tempo) e coeficiente de amortecimento quadrático $(1 / \mathrm{m}$, resistência por unidade de comprimento), $\rho$ é a massa específica, $V$ a velocidade, $Z$ a posição vertical, $Z_{f s}$ e $Z_{b}$ as posições verticais da superfície livre e do fundo, $x$ a posição horizontal, $x_{S}$ e $x_{e}$ as respectivas posições horizontais de início e fim da praia numérica.

Sendo os coeficientes de amortecimento, linear e quadrático, padrões são de 10 resistências por unidade, porém, foi adotado $C_{1}=20$ e $C_{2}=0$ para as simulações realizadas, conforme Lisboa et al. (2016).

\section{Modelo Computacional}

\subsection{Modelo multifásico Volume of fluid (VOF)}

O método Volume of Fluid (Hirt e Nichols, 1981) é um modelo multifásico usado para as soluções de escoamentos compostos de dois ou mais fluidos (ou fases) imiscíveis. Todas as fases são definidas e o volume ocupado por uma fase não pode ser ocupado por outra, para isso o modelo mais apropriado é o VOF. Para cada fase adicionada no modelo, uma nova variável é introduzida, resultando numa fração volumétrica destes fluidos na célula computacional. Para representar as fases contidas em cada volume de controle é necessário o conceito da fração de volume, representado pela letra grega alfa $(\alpha)$.

O modelo utilizado para representar a interação entre duas fases, água e ar, num determinado volume pode ser representado por uma três condições para a fração de volume do fluido: quando $\alpha_{\text {água }}=0$, o volume está completo de ar; quando $\alpha_{\text {água }}=1$, o volume está completo de água e quando $0<\alpha_{\text {água }}<1$, o volume possui as duas fases, neste caso $\alpha_{a r}=1-\alpha_{\text {água }}$.

Assim, a soma em cada célula de todas as fases deve ser igual a um. Ao empregar o modelo VOF, resolve-se um conjunto de equações, a de quantidade de movimento e a de continuidade, que são aplicadas a cada fluido ao longo do volume. A fração de volume de cada fluido em cada célula é considerada em todo o domínio computacional através da equação de transporte para a fração volumétrica. Portanto, o modelo é composto pela equação de continuidade:

$$
\frac{\partial \rho}{\partial t}+\nabla \cdot(\rho \vec{v})=0
$$

a equação da fração volumétrica:

$$
\frac{\partial(\alpha)}{\partial t}+\nabla \cdot(\alpha \vec{v})=0
$$

e as equações de quantidade de movimento:

$$
\frac{\partial}{\partial t}(\rho \vec{v})+\nabla \cdot(\rho \vec{v} \vec{v})=-\nabla p+\nabla \cdot(\mu \overline{\bar{\tau}})+\rho \vec{g}
$$

onde: $\rho$ é a massa específica do fluido $\left(\mathrm{kg} / \mathrm{m}^{3}\right), t$ é o tempo $(\mathrm{s}), \vec{v}$ é o vetor velocidade do escoamento $(\mathrm{m} / \mathrm{s})$, $p$ é a pressão estática (Pa), $\mu$ é a viscosidade $(\mathrm{kg} /(\mathrm{ms}))$, $\overline{\bar{\tau}}$ é o tensor de tensões (Pa) e $\vec{g}$ é a aceleração da gravidade $\left(\mathrm{m} / \mathrm{s}^{2}\right)$.

Uma vez que as equações de conservação de massa e quantidade de movimento são resolvidas para a mistura das fases, é necessário calcular valores médios da massa específica e a viscosidade (Srinivasan et al., 2011):

$$
\rho=\alpha_{\text {água }} \rho_{\text {água }}+\alpha_{a r} \rho_{a r}
$$




$$
\mu=\alpha_{\text {água }} \mu_{\text {água }}+\alpha_{a r} \mu_{a r}
$$

\subsection{Modelo numérico}

Para a solução das equações de conservação da massa e quantidade de movimento, foi empregado um código comercial baseado no Método de Volumes Finitos - MVF (FLUENT). Para todas as simulações foi considerado o esquema de advecção upwind para tratamento dos termos advectivos, enquanto a discretização espacial para a pressão o método PRESTO e para a fração volumétrica o método GEO-RECONSTRUCTION. O método PISO para o acoplamento pressão-velocidade e, para de inicialização de entrada, foi aplicado o método wavy.

Empregaram-se os fatores de sub-relaxação para as equações da conservação da massa de 0,3 e de 0,7 para a quantidade de movimento. Para a solução do sistema de equações gerado após a discretização é utilizado o método Gauss Seidel com multigrid algébrico. Maiores detalhes sobre a metodologia numérica podem ser obtidos em Versteeg e Malalasekera (2007).

Todas as soluções foram realizadas usando computador Intel Core i7 com 6 núcleos e 16.0 Gb de memória RAM. No processamento das simulações foi empregada a técnica do processamento serial disponibilizada pelo software FLUENT ${ }^{\circledR}$. Os softwares GAMBIT (pré-processamento) e FLUENT (pré-processamento, solução e pós-processamento) foram utilizados na modelagem matemática e na solução das equações governantes da simulação numérica.

\section{Resultados e Discussões}

As análises consistem principalmente com base no erro da elevação da superfície livre. Para a análise dos resultados foi realizado uma análise de forma quantitativa e qualitativa. Para comparar as alturas significativas, da solução analítica e numérica, pode-se utilizar a norma $l_{\infty}$, dada por (Kreyszig, 2011):

$$
\left\|l_{\infty}\right\|=\operatorname{má}_{j} x\left|x_{j}\right|
$$

onde, $x_{j}$ representa a variável em consideração instantânea.

O emprego da norma, possibilita analisar a diferença da elevação da superfície livre nos picos de elevação das alturas significativas. Além disso, são empregadas mais duas médias para quantificar as diferenças geradas nas comparações da elevação das superfícies livre das soluções numéricas e analíticas, a média do erro absoluto (MAE Mean Absolute Error), dada por:

$$
M A E=\frac{1}{m} \sum_{i=1}^{m}\left|x_{n u m, i}-x_{\text {ana }, i}\right|
$$

onde, $m$ é o número de amostras analisadas, $i$ é o número da amostra analisada, $x_{n u m, i}$ representa a elevação da superfície livre instantânea obtida numericamente e $x_{a n a, i}$ representa a elevação da superfície livre analítica.

Ainda foi utilizada a média do desvio padrão (RMSE - Root Mean Square Error), dada por:

$$
R M S E=\sqrt{\frac{\sum_{i=1}^{m}\left(x_{n u m, i}-x_{a n a, i}\right)^{2}}{m}}
$$

Para a análise quantitativa, foram consideradas as Eqs. (17) - (19) a fim de calcular a diferença entre as soluções, analítica e numérica, para as doze simulações realizadas em todas as posições das sondas consideradas (Fig. 2).

\subsection{Análise da Discretização Temporal}

O parâmetro de investigação da discretização temporal, o avanço no tempo (time-step), é mais um parâmetro importante na precisão da simulação numérica e no tempo de processamento da simulação, pois a utilização de valores inadequados pode provocar perda de informação, bem como, a não convergência residual da velocidade, da fração de volume e da conservação da massa (Carneiro, 2007).

Nestes testes foi utilizada como recomendação uma malha com 26.320 volumes, obtida através da análise da discretização espacial em Rodrigues et al. (2017), que corresponde ao comprimento mínimo da onda (equivalente a frequência mínima do espectro) com 26.320 volumes. 
Nesse sentido, na Tabela 2, tem-se os valores para o avanço no tempo, onde, foram analisados doze diferentes passos de tempo, em relação ao período de mínimo (referente à frequência mínima do espectro de ondas), ao período de pico (referente à frequência de pico do espectro de ondas) e ao período de máximo (referente à frequência máxima do espectro de ondas).

Tabela 2. Testes relativos à discretização temporal.

\begin{tabular}{ccccc}
\hline Simulação & Avanço no tempo & & Simulação & Avanço no tempo \\
\cline { 5 - 5 } \cline { 5 - 5 } 2 & $T_{\min } / 100$ & 7 & $T_{P} / 500$ \\
2 & $T_{\operatorname{mín}} / 400$ & 8 & $T_{P} / 1000$ \\
3 & $T_{\min } / 500$ & 9 & $T_{\text {máx }} / 100$ \\
4 & $T_{\operatorname{mín}} / 1000$ & 10 & $T_{\text {máx }} / 400$ \\
5 & $T_{P} / 100$ & 11 & $T_{\text {máx }} / 500$ \\
6 & $T_{P} / 400$ & 12 & $T_{\text {máx }} / 1000$ \\
\hline
\end{tabular}

Na Tabela 3, de forma quantitativa, tem-se o cálculo da norma e das médias (MAE e RMSE) através das Eqs. (17)-(19), onde foi considerado a elevação da superfície livre em todas as posições das sondas, ou seja, nas 5 sondas de medição.

Observando os resultados apresentados na Tab. 3, é possível verificar que as diferenças entre as médias e entre as normas (onde, $I_{\text {ana }}$ representa a norma analítica e $I_{\text {num }}$ a norma numérica), em relação a cada sonda, são mínimas. No entanto, ao considerar a diferença de picos, estas diferenças são significativas.

Tabela 3. Análise quantitativa do erro relativo à discretização temporal.

\begin{tabular}{|c|c|c|c|c|c|c|c|c|c|}
\hline & Sonda & MAE & RMSE & $l_{\text {ana }}-l_{\text {num }}$ & & Sonda & MAE & RMSE & $l_{\text {ana }}-l_{\text {num }}$ \\
\hline \multirow{5}{*}{1} & 1 & 0,347651 & 0,446497 & 0,464653 & \multirow{5}{*}{7} & 1 & 0,403444 & 0,517214 & 0,166875 \\
\hline & 2 & 0,315938 & 0,401192 & 0,603350 & & 2 & 0,353696 & 0,443504 & 0,253328 \\
\hline & 3 & 0,301880 & 0,369225 & 0,615339 & & 3 & 0,327366 & 0,413083 & 0,217476 \\
\hline & 4 & 0,310236 & 0,369161 & 0,422366 & & 4 & 0,330647 & 0,402280 & 0,072923 \\
\hline & 5 & 0,312499 & 0,379124 & 0,178869 & & 5 & 0,312356 & 0,376736 & 0,403863 \\
\hline \multirow{5}{*}{2} & 1 & 0,380659 & 0,480173 & 0,210010 & \multirow{5}{*}{8} & 1 & 0,394152 & 0,509195 & 0,147711 \\
\hline & 2 & 0,352573 & 0,442860 & 0,320057 & & 2 & 0,396089 & 0,509153 & 0,214356 \\
\hline & 3 & 0,321055 & 0,396755 & 0,347312 & & 3 & 0,378558 & 0,461741 & 0,194579 \\
\hline & 4 & 0,316611 & 0,379959 & 0,077809 & & 4 & 0,325683 & 0,391734 & 0,072342 \\
\hline & 5 & 0,306729 & 0,372863 & 0,406383 & & 5 & 0,31635 & 0,381109 & 0,403884 \\
\hline \multirow{7}{*}{3} & 1 & 0,360140 & 0,473973 & 0,203155 & \multirow{5}{*}{9} & 1 & 0,438770 & 0,551062 & 0,088578 \\
\hline & 2 & 0,361874 & 0,450723 & 0,291179 & & 2 & 0,384791 & 0,460282 & 0,151573 \\
\hline & 3 & 0,344752 & 0,421128 & 0,301666 & & 3 & 0,385966 & 0,478650 & 0,085874 \\
\hline & 4 & 0,315971 & 0,376417 & 0,077809 & & 4 & 0,278742 & 0,362048 & 0,065805 \\
\hline & 5 & 0,306727 & 0,375234 & 0,40459 & & 5 & 0,425256 & 0,529220 & $-0,109974$ \\
\hline & 1 & 0,328528 & 0,418983 & 0,161198 & & 1 & 0,413171 & 0,523501 & 0,143818 \\
\hline & 2 & 0,319849 & 0,406994 & 0,239473 & & 2 & 0,391742 & 0,478138 & 0,199482 \\
\hline 4 & 3 & 0,299731 & 0,372392 & 0,196585 & 10 & 3 & 0,363564 & 0,435656 & 0,176137 \\
\hline
\end{tabular}




\begin{tabular}{|c|c|c|c|c|c|c|c|c|c|}
\hline & 4 & 0,292378 & 0,364009 & 0,072304 & & 4 & 0,326598 & 0,409090 & 0,071876 \\
\hline & 5 & 0,310065 & 0,377392 & 0,403805 & & 5 & 0,316032 & 0,381353 & 0,404120 \\
\hline \multirow{5}{*}{5} & 1 & 0,352877 & 0,456501 & 0,312468 & \multirow{5}{*}{11} & 1 & 0,395414 & 0,522314 & 0,250615 \\
\hline & 2 & 0,320464 & 0,406456 & 0,440307 & & 2 & 0,397763 & 0,484044 & 0,193715 \\
\hline & 3 & 0,316616 & 0,385656 & 0,464193 & & 3 & 0,350108 & 0,445895 & 0,168422 \\
\hline & 4 & 0,321472 & 0,382195 & 0,235829 & & 4 & 0,357069 & 0,435005 & 0,071705 \\
\hline & 5 & 0,317228 & 0,385314 & 0,097088 & & 5 & 0,317091 & 0,385529 & 0,404424 \\
\hline \multirow{5}{*}{6} & 1 & 0,403936 & 0,506168 & 0,180091 & \multirow{5}{*}{12} & 1 & 0,441795 & 0,554060 & 0,338464 \\
\hline & 2 & 0,355802 & 0,445708 & 0,255215 & & 2 & 0,379333 & 0,464302 & 0,394259 \\
\hline & 3 & 0,307301 & 0,390155 & 0,249198 & & 3 & 0,326277 & 0,400374 & 0,484085 \\
\hline & 4 & 0,327474 & 0,387646 & 0,074861 & & 4 & 0,345772 & 0,440694 & 0,071555 \\
\hline & 5 & 0,308229 & 0,374796 & 0,404073 & & 5 & 0,338863 & 0,399287 & 0,409776 \\
\hline
\end{tabular}

Na Figura 6 tem-se os gráficos da elevação da superfície livre na sonda 1 em relação ao período mínimo da onda, com avanço no tempo de $0,1257 \mathrm{~s}$.

Onde é possível perceber que quanto maior o intervalo do avanço no tempo mais oscilações numéricas ocorrem na elevação da superfície livre. $E$, quanto menor o avanço no tempo, mais tempo é necessário para a simulação numérica começar a repetir o ciclo.

Ao fazer uma comparação da Tab. 3 com a Fig. 6 é possível observar a diferença entre as simulações, em que uma pequena mudança no avanço do tempo pode resultar em consideráveis diferenças entre as simulações, principalmente na diferença entre as normas. Cabe destacar que, na Fig. 6, a elevação da superfície livre analítica não se altera entre os gráficos.
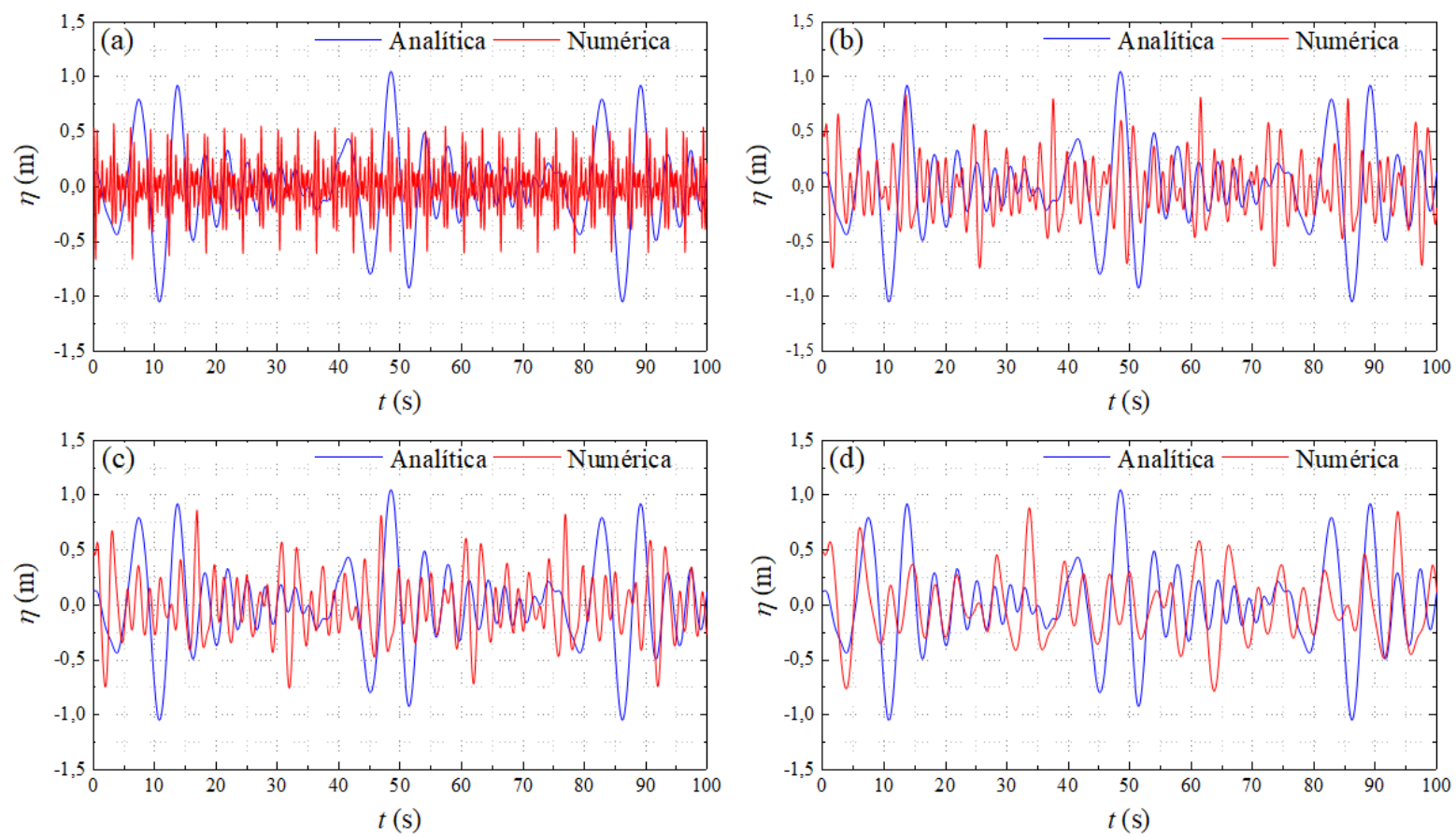

Figura 6. Elevação da superfície livre da discretização temporal na sonda 1 em:

(a) $T_{m i n} / 100$, (b) $T_{\min } / 400$, (c) $T_{\min } / 500$ e (d) $T_{\min } / 1000$. 
Na Figura 6(a), é possível observar que a elevação da superfície livre numérica se repete com mais frequência e as oscilações estão bem próximas. Conforme diminui o passo de tempo, mais tempo a elevação numérica leva para completar o ciclo e as oscilações mais distantes.

Além da análise das normas e médias dos erros, foi realizada uma análise quanto ao tempo de processamento das simulações realizadas, conforme a Tab. 4, pode-se verificar a duração aproximada das simulações, em horas.

Tabela 4. Tempo de processamento.

\begin{tabular}{cc}
\hline Avanço no tempo & Duração da simulação $(\mathrm{h})$ \\
\hline$T_{\text {mín }} / 100$ & 0,90 \\
$T_{\text {mín }} / 400$ & 2,40 \\
$T_{\min } / 500$ & 2,80 \\
$T_{\operatorname{mín}} / 1000$ & 4,42 \\
$T_{P} / 100$ & 1,30 \\
$T_{P} / 400$ & 3,57 \\
\hline
\end{tabular}

\begin{tabular}{cc}
\hline Avanço no tempo & Duração da simulação $(\mathrm{h})$ \\
\hline$T_{P} / 500$ & 4,15 \\
$T_{P} / 1000$ & 6,32 \\
$T_{\text {máx }} / 100$ & 3,12 \\
$T_{\text {máx }} / 400$ & 7,62 \\
$T_{\text {máx }} / 500$ & 9,00 \\
$T_{\text {máx }} / 1000$ & 10,53 \\
\hline
\end{tabular}

Ao considerar os resultados da Tab. 3 e da Tab. 4 e analisando a Fig. 6, tem-se os resultados com maior acurácia para a discretização temporal para $T_{\text {mín }} / 1000$. Onde possui menores erros e diferenças entre as normas e tempo de processamento, mesmo que o modelo subestima as maiores amplitudes e ainda apresenta algumas diferenças de fase.

\section{Conclusões}

Neste trabalho foi apresentada e discutida uma metodologia, disponível no software ANSYS FLUENT (18.1), para a geração numérica de um espectro de ondas do tipo Pierson-Moskowitz. O domínio computacional considerado foi bidimensional e representado por um tanque de ondas numérico com a técnica da praia numérica.

$\mathrm{O}$ espectro de ondas utilizado neste estudo, considera algumas características como período significativo da onda $\left(T_{s}\right)$ de 7,5 s e altura significativa da onda $\left(H_{s}\right)$ de 1,5 m. Foram apresentados diversos detalhes para a simulação numérica de um espectro de ondas. Ainda foram realizadas comparações entre soluções numéricas e analíticas.

Os resultados apresentados indicam que as recomendações estão fundamentadas no período mínimo do espectro. Onde as médias, desvios e as diferenças de picos são melhores em relação aos demais períodos quando comparados com o tempo de processamento, caracterizando assim, o avanço do tempo de $T_{\operatorname{mín}} / 1000$ como recomendação. Os resultados obtidos indicam que esta técnica pode ser empregada com sucesso, representando assim um avanço na simulação numérica de estruturas oceânicas, pois, é possível simular computacionalmente em tanques de ondas o fenômeno muito próximo do real.

O modelo ainda possui algumas dificuldades em representar as maiores frequências e em manter os sinais totalmente em fase. Assim, é necessário que o modelo seja aperfeiçoado em questões como: i) considerar o regime turbulento, ii) considerar profundidades maiores, iii) diferentes números de componentes de ondas, iv) influência do número de pontos na análise por FFT.

Agradecimentos: Os autores agradecem ao Instituto Federal do Paraná (IFPR), à Universidade do Vale do Rio dos Sinos (UNISINOS), à Universidade Federal de Rio Grande (FURG) e ao CNPq pelo suporte financeiro

\section{Referências}

1. Ansys. Theory Guide. FLUENT 2016.

2. Anbarsooz, M.; Fard, M. P.; Moghiman, M. (2013). Fully nonlinear viscous wave generation in numerical wave tanks. Journal of Ocean Engineering, 59, 5973-85.

3. Boo, S. Y. (2002). Linear and nonlinear irregular waves and forces in a numerical wave tank. Journal of Ocean Engineering, 29, 475-493.

4. Carneiro, M. L. (2007). Desenvolvimento de dispositivo de geração e absorção ativa de ondas para tanque de ensaio de estruturas oceânicas. Dissertação (Mestrado, Escola Politécnica da Universidade de São Paulo, São Paulo - SP, 116 p. 
5. Chakrabarti, S. K. (2005). Handbook of offshore engineering. Elsevier, 1, $661 \mathrm{p}$ Illinois, Estados Unidos.

6. Cho, H. Y.; Kweon, H. M.; Jeong, W. M. (2015). A study on the equation of the continuous wave spectrum. International Journal of Naval Architecture and Ocean Engineering, 7, 1056-1063.

7. Costa, A. O.; Santos, E. D.; Isoldi, L. A.; Rocha, L. A. O.; Gomes, M. N. (2016). Simulação numérica de um espectro de ondas do tipo Pierson - Moskowitz. VII SEMENGO - Seminário e Workshop em Engenharia Oceânica. Rio Grande, p. 258-266.

8. Dean, R. G., Dalrymple, R.A. (1991). Water wave mechanics for engineers and scientists. World Scientific, 2, 353 p. Singapura.

9. Elangovan, M.; Sahoo, G. (2010). Generation of Irregular Wave for Marine Application by Plunger Type Wave Maker. $11^{\text {th }}$ International Symposium on Practical Design of Ships and Other Floating Structure, Rio de Janeiro, RJ, Brazil.

10. Elangovan, M. (2011). Simulation of irregular waves by CFD. World Academy of Science, Engineering and Technology, 5, 427-431.

11. Finnegan, W.; Goggins, J. (2015). Linear irregular wave generation in a numerical wave tank. Applied Ocean Research, 52, 188-200.

12. FLUENT 17.0. Documentation Manual, 2017. Disponível em:

13. <http://www.ansys.com/Products/Fluids/ANSYS-Fluent>.

14. Gatin, I.; Vukcevic, V.; Jasak, H. (2017). A framework for efficient irregular wave simulations using higher Order Spectral method coupled with viscous two phase model. Journal of Ocean Engineering and Science, 2, 253-267.0

15. Gomes, M. N.; Costa, C. A. O.; Deus, M. J.; Ricardo, R. L. P.; Isoldi, L. A.; Santos, E. D.; Rocha, L. A. O. (2016). Análise computacional e geométrica com design construtal de um dispositivo conversor de energia das ondas do mar em energia elétrica do tipo coluna de água oscilante submetido a um espectro de ondas. In: Proceedings XXXVII Iberian Latin American Congress on Computational Methods in Engineering (CILAMCE 2016), Brasília, Brazil.

16. Hirt, C.W.; Nichols, B.D. (1981). Volume of fluid (VOF) method for the dynamics of free boundaries. Journal of Computational Physics, 39(1), 201-225.

17. Kamath, A.; Bihs, H.; Arntsen, O. A. (2015). Numerical investigations of the hydrodynamics of an oscillating water column device. Journal of Ocean Engineering, 102, 40-50.

18. Kim, M. W.; Koo, W.; Hong, S. Y. (2014). Numerical analysis of various artificial damping schemes in a three-dimensional numerical wave tank. Journal of Ocean Engineering,75, 165-173.

19. Kreyszig, E., Kreyszig, H., Norminton, E. J. (2011). Advanced Engineering Mathematics. 10a ed., 1283 p.

20. Liang, X. F.; Yang, J. M.; Li, J.; Xiao, L.; Li, X. (2010). Numerical simulation of irregular Wave-Simulating Irregular wave train. Journal of Hydrodynamics, Ser. B, 22, 537-545.

21. Lisboa, R.; C.; Teixeira, P. R. F.; Didier, E. (2016). Simulação da propagação de ondas regulares e irregulares em um canal bidimensional com praia numérica. VII SEMENGO - Seminário e Workshop em Engenharia Oceânica, Rio Grande, p. 258-266.

22. Park, J. C.; Uno, Y.; Sato, T.; Miyata, H.; Chun, H. H. (2004). Numerical reproduction of fully multi-directional waves by a viscous 3D numerical wave tank. Journal of Ocean Engineering, 31, 1549-1565.

23. Prasad, D. D.; Ahmed, M. R.; Lee, Y. O.; Sharma, R. N. (2017). Validation of a piston type wave-maker using Numerical Wave Tank. Journal of Ocean Engineering, 131, 57-67.

24. Rezanejad, K.; Guedes S. C.; López I.; Carballo R. (2017). Experimental and numerical investigation of the hydrodynamic performance of an water oscillating column wave energy converter. Renewable Energy, 106, 1-16.

25. Rodrigues, L.; Oliveira, S. S.; Dos Santos, E. D.; Isoldi, L. A.; Rocha, L. A. O.; Gomes, M. N. (2017). Análise da discretização espacial para a simulação numérica de um espectro de ondas do tipo Pierson - Moskowitz. In: Iberian Latin American Congress on Computational Methods in Engineering (CILAMCE, 2017), Florianópolis, Brazil.

26. Srinivasan, V.; Salazar, A.J.; Saito, K. (2011). Modeling the disintegration of modulated liquid jets using volume of fluid (VOF) methodology. Applied Mathematical Modeling, 35(8), 3710-3730.

27. Versteeg, H. K.; Malalasekera, W. (2007). An Introduction to Computational Fluid Dynamics - The Finite Volume Method. Pearson, England.

28. Vyzikas, T.; Deshoulières, S.; Giroux, O.; Barton, M.; Greaves, D. (2017). Numerical study of fixed oscillating water column with rans-type two-phase CFD model. Renewable energy, 102(b), 294-305. 
29. WMO (1998). Guide to wave analysis and forecasting. Livro World Meteorological Organization, GenevaSwitzerland, $2^{\circ}$ edição.

30. Zhang, X. T.; Khoo, B. C.; Lou, J. (2006). Wave propagation in a fully nonlinear numerical wave tank: A desingularized method. Journal of Ocean Engineering, 33, 2310-2331. 